rev.relac.int.estrateg.segur.10(2):215-235,2015

\title{
LA DIPLOMACIA CULTURAL EN LOS NEGOCIOS INTERNACIONALES: RELIGIONES DEL MUNDO*
}

\author{
Henry Acuña Barrantes ${ }^{* *}$ \\ Pedro Nonell Torres ${ }^{* * *}$
}

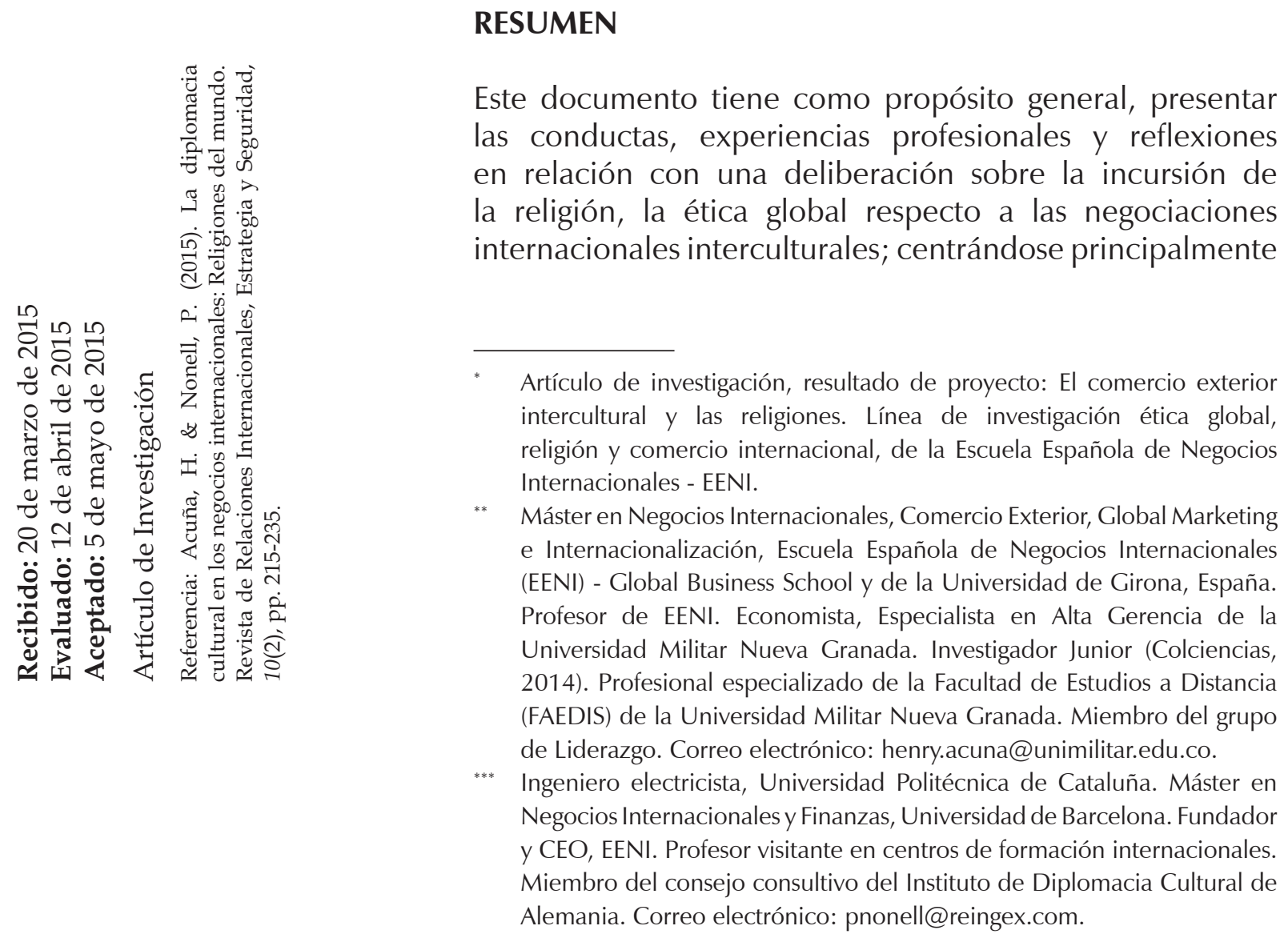


en el lado positivo y tangible de las religiones y la responsabilidad social corporativa (RSC); fue perfilado bajo el paradigma cualitativo y el enfoque hermenéutico. Se utilizó el método documental y descriptivo; el primero, por la consulta realizada a diferentes documentos científicos en internet; y el segundo porque de la información encontrada en la web se extrajo, describió e interpretó lo más trascendente de determinada culturas, personalidades y organizaciones. Teniendo presente que quienes conocen una sola representación cultural, argumentan que podrían tener un conocimiento de las características culturales de otros lugares, cuando en realidad no es así. Siglos atrás las religiones ya ofrecieron normas de conducta y valores para que las personas implementen en su diario vivir y más aún al libre comercio local e internacional. Por esta razón las personas de diferentes dogmas (Hindú, Cristiana, Islámica, Budistas, etc.) desean conocer cómo implementar sus creencias para el entorno empresarial para una mejor comprensión entre las partes y crear sinergias entre ellas (religión- cultura - comercio).

Palabras clave: Comunicación intercultural, Cultura, Ética Global, Negocios Internacionales, Religión.

\title{
CULTURAL DIPLOMACY IN INTERNACTIONAL BUSINESS: WORLD RELIGIONS
}

\begin{abstract}
This document general purpose is to present behavior, professional experiences and reflections of characters to make a deliberation on the incursion of religion, global ethics in relation to cross-cultural international negotiations; mainly focusing on the positive and tangible side of the religious and corporate social responsibility (CSR); it was shaped under the qualitative paradigm and the hermeneutic approach. The method is documentary and descriptive. the first one, by the consultation made to different scientific documents on the internet; and the second one because of the information found on the web was extracted, described and interpreted the most transcendent of specific cultures, personalities and organizations. Bearing in mind that those who know a single cultural representation, argue that they could have a knowledge of other cultural characteristics, when in reality it is not so. Centuries ago the religions already offered standards of conduct and values people implement in their daily living and even to the local and international free trade. For this reason people of different dogmas (Hindu, Christian, Islamic, Buddhists, etc.) want to know how they can implement their beliefs for the business environment for a better understanding between the parties and create synergies between them (religion-culture-trade).
\end{abstract}

Keywords: Intercultural Communication, Culture, Global Ethics, International Business, Religion. 


\section{DIPLOMACIA CULTURAL, NEGÓCIOS INTERNACIONAIS: RELIGIÕES DO MUNDO}

\section{RESUMO}

Este documento é de uso geral, para apresentar comportamento, experiências profissionais e reflexões fazendo uma deliberação sobre a incursão de religião, ética global em relação às negociações internacionais transculturais; principalmente com foco no lado positivo e tangível dos religiosos e da Responsabilidade Social Corporativa (RSC); Ela foi moldada sob o paradigma qualitativo e a abordagem hermenêutica. O método descritivo e documental. O primeiro, por consulta feita aos diferentes documentos científicos na internet; e o segundo por causa da informação encontrada na web foi extraído, descrito e interpretado o mais transcendente de organizações, personalidades e culturas específicas. Tendo em conta que quem conhece uma única representação cultural, argumentam que eles poderão ter um conhecimento de outras características culturais, quando na realidade não é assim. Há séculos as religiões já ofereceram as normas de conduta e as pessoas de valores implementam em sua vida diária e até mesmo para o livre comércio local e internacional. Por esta razão as pessoas de diferentes dogmas (hindu, cristã, islâmica, budistas, etc.) quer saber como eles podem implementar suas crenças para o ambiente de negócios para um melhor entendimento entre as partes e criar sinergias entre eles (comércio de religião-cultura-).

Palavras-chave: Comunicação Intercultural, Cultura, Ética Global, Negócios Internacionais, Religião.

\section{INTRODUCCIÓN}

"Todo el mundo debe seguir su propia religión. Un cristiano debe seguir el cristianismo; un musulmán debe seguir el Islam y así sucesivamente" (Sri Ramakrishna, 1903).

En un acuerdo comercial participan dos o más partes, buscando alcanzar un resultado satisfactorio dentro del mercado. Para alcanzarlo tienen que resolverse los conflictos o contratiempos que puedan surgir al momento de negociar. Lo anterior no es labor sencilla porque, en muchas ocasiones, alguna de las partes no está dispuesta a ceder, por lo que es necesario planear estrategias, así como el obtener la mayor cantidad de información posible para entablar una adecuada comunicación que favorezcan a uno y otro.

Sin embargo, un aspecto indispensable que se tiene que prever y tener en cuenta antes de hacer cualquier negociación es el aspecto ético, religioso, social, cultural, ambiental, entre varios elementos. No hay una sola nación que pueda considerarse autosuficiente por sí misma y que no necesite del concurso y apoyo de los demás; aun los países más ricos demandan recursos de los cuales carecen y que por medio de las negociaciones y acuerdos mundiales 
suplen sus necesidades y carencias en otras zonas, esto se debe a que no todos los países negocian de la misma manera.

El objeto de este documento es analizar cómo influye la cultura, la ética y sumado con la interacción de la religión en el contexto intercultural en las negociaciones internacionales, cómo las grandes personalidades y organizaciones están presenten en el mundo interactuando según su convicción, centrándose principalmente en el lado positivo y tangible de las religiones y la responsabilidad social corporativa (RSC) que implementan algunas organizaciones en el mundo.

El presente artículo se encuentra elaborado bajo el paradigma cualitativo y el enfoque hermenéutico. Se utilizó el método documental y descriptivo; el primero, por la consulta realizada a diferentes documentos científicos en internet; y el segundo porque de la información encontrada en la web se extrajo, describió e interpretó lo más trascendente de determinada culturas, personalidades y organizaciones.

Siguiendo el pensamiento de Dudley Seers (1970), referente a la incorporación de factores de carácter social para explicar el crecimiento económico, inspirado en el pensamiento de Gandhi, plantea que para que exista desarrollo deben darse condiciones que garanticen la realización del potencial humano, sintetizándolas en alimentación, empleo y no discriminación, así entonces, considera de manera formal el efecto que sobre la economía tiene la religión.

Welch (2001) argumenta que la economía está en servicio de la religión, así entonces las creencias religiosas afectan las preferencias de las personas y por lo tanto modifican el comportamiento del mercado, terminando éste último, actuando en base a lo aceptado por la religión. En palabras de la página Redes cristianas (2006):

Seis de los 10 países con la población más rica del mundo, según datos de la CIA, tienen al protestantismo como culto principal. Del resto, uno la musulmana y tres la católica. Teólogos explicaron la relación entre el dinero y el culto.

Luxemburgo, Irlanda y San Marino son las tres naciones con mayoría católica de la lista de los 10 países con la población más rica del mundo, elaborada por la Central de Inteligencia (CIA) de los Estados Unidos a julio de 2006, en base a la Paridad del Poder de Compra per cápita (PPP).

Salvo por Emiratos Árabes Unidos, cuya población profesa en 96 \% el culto musulmán, el resto sostiene la religión protestante, luterana, calvinista, evangélica pentecostal o anglicana.

Otros autores como el caso de Barros \& Garoupa (2002) han incursionado al estudio de la economía y la religión bajo el análisis de una estructura de mercado, considerando las diferentes opciones religiosas como la oferta de mercado, y la afiliación a alguna de ellas como 
la demanda, y tratando de explicar porque algunas tienen mayor número de demandantes en base a una elección de los adherentes de acuerdo con los diferentes criterios de exigencia de cada religión.

Es importante conocer de acuerdo con Rugman (2000), los aspectos esenciales de una negociación internacional, las definiciones y las partes que intervienen en ella, en últimas, los aspectos que comúnmente se negocian, así como las etapas por las cuales la mayoría de las negociaciones internacionales atraviesan, es decir, el esquema general de la negociación.

La negociación la describe Ball (1997) como el proceso mediante el cual las partes involucradas, donde una ostenta o puede hacer algo que la otra anhela y viceversa, se comunican recíprocamente con el objetivo de lograr un acuerdo que brinde las bases para un correcto entendimiento que ayude a la consecución de las necesidades y por lo tanto la satisfacción de las partes interesadas. A su vez, Pruitt, (1986) lo describe como un proceso en el que se toma una decisión conjunta por dos o más partes. Las partes verbalizan en primer lugar sus demandas contradictorias, moviéndose posteriormente hacia el acuerdo mediante un proceso de realización de concesiones o búsqueda de nuevas alternativas.

El arte de la negociación no es señal exclusiva de nuestro tiempo, estos lineamientos ya se aseveraban tres siglos atrás, así entonces se debe proveer y contemplar. En la obra francesa de 1716 "De la Maniére de Négocier avec les Souverains", se extrae el siguiente argumento:

El diplomático debe ser rápido, recursivo, buen oyente, cortes y agradable. No debe crearse la reputación de ser un talento, ni tampoco debe discutir en exceso de forma que divulgue información secreta para ganar una discusión. Un buen negociador debe sobre todo dominarse suficientemente para resistir la tentación de hablar antes de haber pensado a fondo lo que se propone ceder. No debe caer en el error de suponer que un poco de misterio dentro del cual se crean secretos de la nada y se elevan las trivialidades al nivel de los asuntos de estado, es algo más que el síntoma de una cabeza chica. Deberá prestarle atención a las mujeres pero sin entregar su corazón. Deberá ser capaz de aparentar dignidad aun sin poseerla, pero al mismo tiempo tendrá que evitar cualquier exhibición de mal gusto. El ser valiente una cualidad esencial puesto que el tímido no podrá esperar culminar con éxito una negociación confidencial.

El negociador deberá tener la paciencia del relojero y carecer de prejuicios personales. No debe tener inclinación por la bebida, el juego o las mujeres, ni ser irritable, ni dejarse llevar por otras veleidades y fantasías. El negociador deberá además estudiar historia, conocer las instituciones extranjeras y las costumbres y ser capaz de decir, para cualquier país extranjero, en manos de quien realmente reside la soberanía. Deberá tener también algún conocimiento de la literatura, ciencia, matemáticas y derecho. Finalmente deberá ser buen anfitrión. Un buen cocinero es a menudo un excelente conciliador. 
Así entonces, en lo referente a los negocios internacionales, son todas las actividades comerciales públicas o privadas que involucran a dos o más países. Las compañías privadas realizan estas transacciones con afán de lucro; los gobiernos pueden o no perseguir lo mismo en sus respectivas transacciones. El profundo conocimiento de los negocios internacionales le permitirá a cualquiera de las dos, tomar decisiones más informadas, como las referentes al lugar en el que desea trabajar y las políticas gubernamentales que le parecen aceptables y sus costumbres éticas, sociales o religiosas.

En lo referente con la religión, palabra del latín religare o re-legere ${ }^{1}$, ha sido y es motivo de discusión, el sociólogo Lenski (1967) exterioriza "es un sistema compartido de creencias y prácticas asociadas, que se articulan en torno a la naturaleza de las fuerzas que configuran el destino de los seres humanos". Según lo describe Clifford Geertz (1973) planteando una definición alternativa:

La religión es un sistema de símbolos que obra para establecer vigorosos, penetrantes y duraderos estados anímicos y motivaciones en los hombres, formulando concepciones de un orden general de existencia y revistiendo estas concepciones con una aureola de efectividad tal que los estados anímicos y motivaciones parezcan de un realismo único.

De acuerdo a su condición de entender y aceptar a la divinidad, existen distintas tipologías de religiones como por ejemplo; la monoteísta que se fundamentan en la presencia de un único Dios, creador de todas las cosas (como el cristianismo, el judaísmo y el islamismo). La politeísta, en cambio, sustenta que existen múltiples dioses, los cuales pueden situarse en un cierto orden jerárquico (como el hinduismo). Asimismo puede hablarse de los cultos panteístas que afirman que el creador y los objetos creados forman una misma entidad (como el taoísmo) y de las religiones no-teístas que no creen en divinidades de poder ilimitado o universal (como el budismo).

Por otra parte, la religión tiene en la actualidad un pergamino significativo en cualquier negociación, más con países africanos y orientales, aún en la mayoría de organizaciones que maniobran el área de comercio internacional, no se evidencia su transcendencia a pesar de que según Nonell (2013) "El 84 por ciento de la humanidad aproximadamente 6.000 millones de personas afirman pertenecer a alguna religión"; el 16 por ciento restante son no creyentes, allí se incluyen: ateos, agnósticos y toda aquel individuo que no se identifican con ninguna religión en particular, es por lo anterior que las que sí, están tomando ventaja a las demás, y la religión como factor importante en el comercio mundial intercultural ${ }^{2}$.

1. Etimología que da Cicerón del término que significa "estar atento, considerar y observar, mantenerse unidos".

2. Definido como un proceso de comunicación e interacción entre personas y grupos con identidades culturales específicas, donde no se permite que las ideas y acciones de una persona o grupo cultural esté por encima del otro, favoreciendo en todo momento el diálogo, la concertación y con ello, la integración y convivencia enriquecida entre culturas. 


\section{LA ÉTICA GLOBAL-CULTURAL ${ }^{3}$ EN EL COMERCIO}

Por otra parte Gelles (2000) detalla la cultura como la suma de las creencias, reglas, técnicas, instituciones e instrumentos que caracterizan a los grupos humanos. En otras palabras, la cultura consiste en patrones comunes de comportamiento que se aprenden de los miembros de una determinada sociedad, lo cual representa el estilo particular de vida de un grupo específico de persona. Ahora bien, el papel que la cultura deben desempeñar en la búsqueda de una ética global es aún más complejo, a menudo la cultura es percibidas como sistemas homogéneos de ideas y creencias. De ahí que la gente se refiera frecuentemente a las culturas china, africana, islámica u occidental, como si las ideas de cada una de ellas constituyesen un todo. Sin embargo, esta percepción deberá ser precisada de varias maneras; porque algunos piensan erradamente que quien conoce una sola representación cultural, argumentan que podrían tener un conocimiento de las características culturales de otros territorios, cuando en realidad no es así; al menos que hayan conseguido hacer comparaciones con otras culturas, tal vez ni siquiera conozcan las características más importantes de la suya.

También sobreviene el hecho de que cada sociedad (Molero, 2006) considera que su cultura es superior a la de los demás y que sus intentos por introducir su forma encuentren una resistencia no deseada. Por lo anterior, el primer paso es comprender que existen culturas diferentes, religiones distintas a la propia.

Actualmente, las organizaciones que irrumpen en los negocios internacionales para maniobrar eficazmente, sus funcionarios u áreas de comercio dentro de la compañía deben conocer las técnicas de comercio exterior (aduanas transporte, Incoterms, etc.), de marketing (distribución, precios, adaptación de productos, políticas de fomento, entre otras); el entorno de los mercados donde pretende hacer las transacciones, así como los fundamentos de la negociación intercultural. Asimismo, se deben estudiar e incluso investigar los principios éticos y religioso, de donde se realizará el negocio para obtener una mayor probabilidad de éxito.

En función de lo anterior, en cualquier negociación se pueden vislumbrar numerosos comportamientos, o actitudes entre culturas, de estos nos exterioriza el autor, Aureoles (2012):

Cuando se negocia con alemanes se espera que sean lógicos a la vez que poco flexibles; de los franceses un cierto aire de superioridad; de los ingleses un trato frío aunque no exento de sentido del humor. Cuando se negocia en Estados Unidos se espera cierta agresividad, trato informal y agilidad en la decisiones, prácticamente lo contrario que en Japón. De los latinos se espera un trato cordial y mucha incertidumbre sobre el resultado de las negociaciones. En los países árabes, hospitalidad y una negociación centrada en el regateo del precio.

3. Definida como experiencias que percibe el ser humano en el presente y lo que experimentarán en el futuro y su entorno. 
Ahora bien, todos los representantes involucrados en la negociación internacional, (empresarios, inversionistas, acreedores, trabajadores, consumidores, entre otros.) tienen una responsabilidad común y esencial, junto con las instituciones políticas y las organizaciones internacionales, la cual es velar por un presente en armonía para todos los seres vivientes, y en un futuro donde la humanidad pueda mantener y se obligue en velar por el bienestar del planeta; para que esto sea posible, dependerá de la capacidad de aprendizaje de los pueblos y los gobiernos en reconocer que la principal manera de servir a los intereses de la humanidad como un todo, consiste en aceptar un acumulado de derechos y responsabilidades para todos.

\section{CREENCIAS Y AFILIACIONES RELIGIOSAS}

Las creencias y afiliaciones religiosas ennoblecen las vidas de más del 84 por ciento de la población mundial ${ }^{4}$; es complejo demostrar plenamente con números exactos debido a que no todas las organizaciones religiosas, o grupos creyentes mantienen registros de sus devotos.

Con relación a la religiosidad, en palabras propias de Sri Ramakrishna (1903) nos expone:

Dios ha hecho diferentes religiones para adaptarse a las diferentes aspiraciones, tiempos y países... todas las doctrinas son sólo tantos caminos; Pero el camino no es Dios mismo.... Todo el mundo debe seguir su propia religión. Un cristiano debe seguir el cristianismo; un musulmán debe seguir el Islam y así sucesivamente.

De lo anterior, se exteriorizan algunos de los cultos más representativos, por número de seguidores, e importancia en que ellas han repercutido sus líderes en el comercio mundial (ver Figura 1).

Hinduismo. Es una composición de religiones que no tiene un fundador único o una autoridad central y la practican más del 80 por ciento de la población en India (estimada para el 2013 en 1.243.000.000 personas).

Confucianismo. Se considera al confucianismo como religión, ya que Confucio desarrolló la filosofía con base en la noción de que toda la realidad está sujeta a un mandato eterno desde el cielo; no creía en la vida después de la muerte.

Taoísmo. Que representa "filosofía del camino", sustenta que cada persona refleja las mismas fuerzas, las energías masculinas y femeninas (yin y yang), que gobiernan el cosmos.

4. Según indica la Asociación de los Archivos de Religión. 
Figura 1. Religiones del mundo

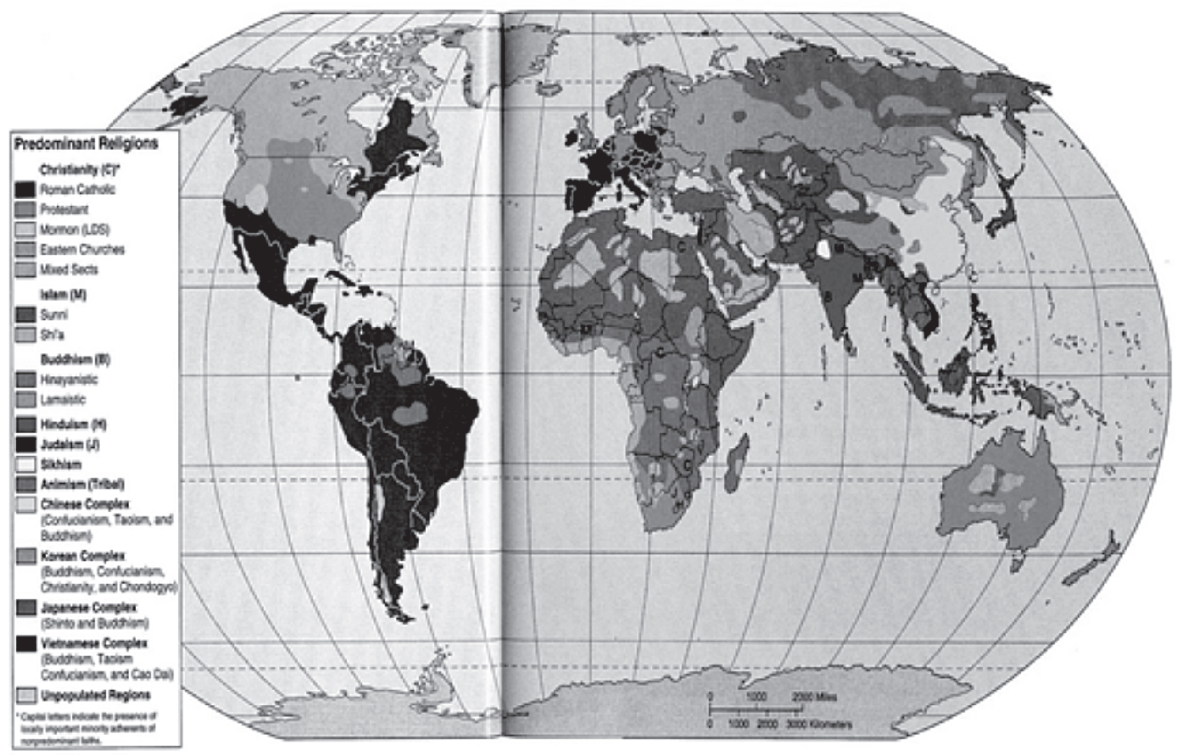

Fuente: Princeton (s.f.).

Islam. Son aproximadamente 1.000 millones de discípulos que hacen de esta joven e universal religiosidad la tercera más grande del mundo, después del cristianismo e hinduismo. El Islam significa "someterse" en árabe; y musulmán, significa "sometimiento". Esta convicción toma el Corán como la palabra eterna de Dios. Los deberes espirituales básicos de todos los musulmanes constan de los cinco pilares de la fe:

- Aceptar la confesión de fe (No hay más Dios que Alá y Mahoma es su profeta).

- Rezar cinco veces al día las oraciones con el rostro hacia La Meca.

- Dar caridad.

- Ayunar durante el día todo el mes de Ramadán (un mes de 29 ó 30 días en el calendario lunar islámico) y

- Hacer un peregrinaje a La Meca al menos una vez en la vida.

Cristianismo. El cristianismo enseña que existe un único Dios en toda la existencia (monoteísta), el cual hizo el universo, la tierra y creó a Adán y a Eva. Dios creó al hombre a su imagen. Existen aproximadamente 2000 millones de creyentes en el mundo.

Jainismo. Es una religión de la India, creada en el siglo VI a. C. por Majavirá. Él decía ser el último omnisciente de una serie de maestros iluminados llamados tirthankaras. La UNESCO en el año 2013 declaró las escrituras Jain "Shantinatha Charithra" como un interés y un valor para la humanidad, por sus contribuciones a la paz, la no-violencia y la fraternidad. 
El principio ético fundamental de Janis es la práctica de la no-violencia o Ahimsa. De este principio fundamental emanan un conjunto de principios éticos:

- No matanza -no-violencia (Ahimsa) "para ser libre de lesiones".

- No mentir - veracidad (Satya) "para ser libre de la mentira".

- No hurtar - justicia (Asteya) "para ser libre de robo".

- Renuncia a lo material (desprendimiento) "está libre de apego mundano".

- A estar libre de impurezas (Brahmacharya). "Para ser libre de fornicación".

- No tener sexo ilícito. En el caso de los monjes también castidad

El mayor pecado que puede hacer un Jain es causar dolor a un ser vivo, hombre o insectos. También previenen daños a la tierra, viento o piedras.

Derivado del principio de la no violencia y su práctica activa, los Jainistas son casi exclusivamente vegetarianos y a menudo practican ayunando. Tal vez la rectitud moral fuerte y honestidad tienden a ser una característica de este culto en los negocios.

Sijismo. La doctrina básica del Sijismo consiste en la creencia en un único Dios y en las enseñanzas de los diez gurús del Sijismo, recogidas en el libro sagrado de los sijes, el Gurú Granth Sahib. Es una religión india fundada por Gurú Nanak

Principios éticos de los Sikhs (Llamado a los seguidores del sijismo)

- La igualdad de la humanidad. Igualdad entre hombres y mujeres

- Moralidad y rectitud, moderación de las pasiones y deseos.

- No robar

- Honestidad

- La filantropía - Justicia Social. El 10 por ciento de sus beneficios deben ser donados a caridad.

- Justicia. Siempre defender a los débiles y oprimidos

Zoroastrianos. El zoroastrismo o mazdeísmo es el nombre de la religión y filosofía basada en las enseñanzas del profeta y reformador iraní Zoroastro (Zarathustra), que reconocen como divinidad a Ahura Mazda (Dios principal), considerado por Zoroastro como el único creador increado de todo.

Las múltiples religiones relacionadas y su interacción intercultural entre la religión y los negocios lo señala Lee (2013). Él quería establecer sí una religión común podía jugar un papel similar en el impulso del comercio, para ello comparó socios comerciales, evaluando si ambos países compartían una religión dominante, descubrió que las naciones que conllevan una 
religión, ya sea a nivel institucional o a nivel de la red, tenían un comercio significativamente mayor, incluso descontando otros efectos (como el lenguaje, nivel cultura, ambiental, etc.); Lee concluye que "la religión establece las redes co-religiosas que afectan positivamente a la confianza interpersonal, reduciendo en consecuencia las distancias institucionales entre países" (2013). El estudio de Lee, refuerza el pensar que la confianza es más importante cuando las culturas se conocen y se comparten.

\section{ORGANIZACIONES QUE DINAMIZAN SU CREENCIA}

Figura 2. Áreas económico-religiosas-culturales

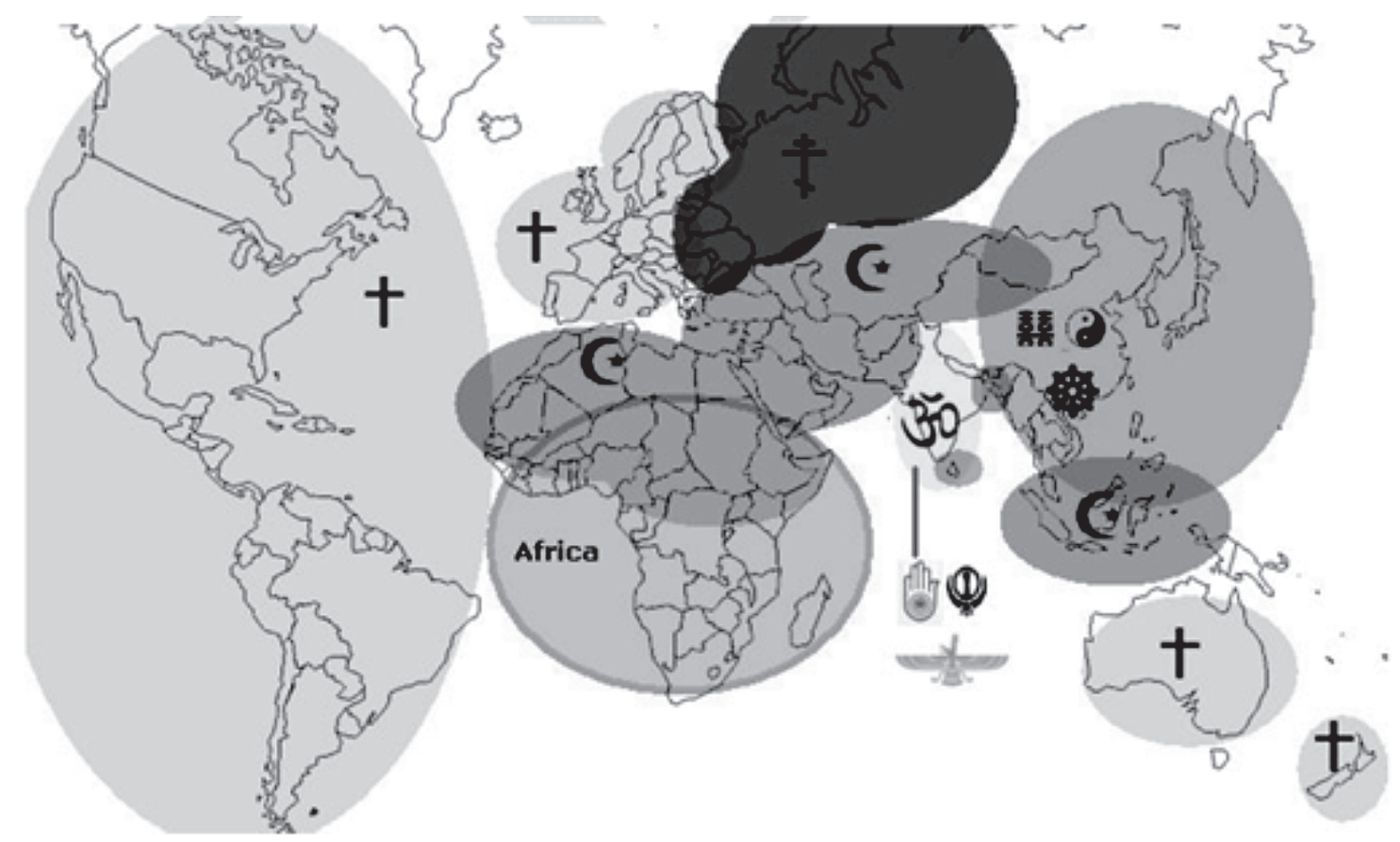

Fuente: EENI (s.f.)

Se presentan las organizaciones económico-religiosas-culturales, en donde la regionalización puede estar desplazando en algunos aspectos a la globalización. Lo anterior, se observa en las seis grandes áreas que son civilizaciones lideradas normalmente por un estado central.

1. La islámica, con Arabia Saudí como estado central

2. La sínica, con China

3. La hindú, con la India

4. La ortodoxa, con Rusia

5. La occidental, con Estados Unidos y la Unión Europea.

6. La civilización africana, con Nigeria y/o África del Sur. 
Otras áreas o quizá sub-civilizaciones que pueden surgir en el mediano plazo son la Japonesa y la Latinoamericana. En función de lo anterior, cada una de estas zonas en cabeza de sus líderes y las organizaciones están explorando, desarrollando e incluso financiando sin fines de lucro, sus enseñanzas, afiliaciones y creencias religiosas en beneficio de la humanidad y del comercio exterior; estas comparten una preocupación por el bienestar del planeta y sus habitantes.

Existen muchos ejemplos de organizaciones empresariales que, además de sus gestiones de proporcionar puestos de trabajo, han dispuesto actividades relativas a la sostenibilidad en sus comunidades; inclusos algunas custodian el medio ambiente y la aplicación de la RSC. De lo anterior, se relacionan tres empresas que aplican la filantropía y fueron materia de este documento.

CIPLA Laboratories, es una prominente compañía farmacéutica india, más conocida fuera de su país de origen, por la fabricación a bajo costo de medicamentos contra el SIDA para pacientes VIH positivos para los países en desarrollo, su presidente Yusuf Khwaja Hamied (Yuku), filántropo ${ }^{5}$ y empresarios en la India (Musulmán); es una persona filantrópica y modelo a seguir de equilibrio entre el negocio, objetivos humanitarios y sociales. El doctor Hamied señala "no quiero ganar dinero con estas enfermedades que causan mucho daño a la sociedad". CIPLA fue el primer laboratorio en ofrecer un paquete de droga triple, Triomune, que puede transformar la vida de los pacientes con VIH; gracias a la visión del doctor Hamied, el costo del tratamiento SIDA cayó dramáticamente de US\$12.000 por paciente, a US\$300 dólares por año.

Grupo Evergreen, se ha ampliado más allá de la industria naviera abarcan operaciones en fuerte desarrollo industrial, servicios de transporte, hoteles y resorts de aire, con más de 27.000 empleados y más de 220 oficinas y agentes en todo el mundo, su presidente el doctor Chang Yung-fa de origen taiwanés expresa:

El dinero es algo que debe ser distribuido en todo el mundo y disfrutado no sólo por una persona... Muchas personas piensan que ganar mucho dinero es riqueza. Para mí, trabajar duro para ganar mucho dinero sin duda es una cosa mala, porque la alegría de ganar mucho dinero es fugaz por el dolor que sientes cuando pierdes. Pero si ese dinero se utiliza para realizar buenas obras, es maravilloso ver los beneficios de lo que has dado. Que felicidad obtenida haciendo una buena obra estará siempre en el corazón.

5. Es el individuo que se caracteriza por el amor hacia las demás personas y sus obras por el bien común sin recibir nada a cambio. El término filántropo es de origen griego "philos o filos" que significa "amor" y "anthropos" que indica "hombre", por lo tanto, la unión de ambas palabras representa "amor al hombre". En referencia a lo anterior, la filantropía es un sentimiento que consiste en amar al género humano sin fines de lucro o solicitar nada a cambio. La Academia de Platón indica que filantropía es un estado de buenas conductas emanadas por el amor a la humanidad. 
Del aparte anterior, se evidencia una clara inspiración de la religión budista. En febrero de 2012, Chang informó que se había comprometido a regalar toda su riqueza (1,69 mil millores de dólares) a la Fundación de Chang Yung-fa. Él pertenece a "I Kuan Tao IKT" (Yiguan Tao) religión, lanzada en China en el siglo pasado; es una religión sincrética, incorporando elementos del taoísmo, el confucianismo y el budismo. El "I Kuan Tao" reconoce a otras religiones como el hinduismo, el Islam o el cristianismo como religiones válidas.

Bennett, Coleman \& Co. - Presidente Indu Jain (jainista), pertenece a la familia de Sahu Jain, posee el más grande grupo de medios de comunicación, posee el Times of India (el periódico más leído en el mundo) y otros periódicos importantes. Creó la Fundación para la filantropía "Times". La señora Indu Jain dirigió un discurso en la Cumbre de las Naciones Unidas en el año 2000, en el que insistió en la necesidad de unidad entre las religiones del mundo. Ella es partidaria del movimiento para difundir los principios de la paz mundial.

\section{EL PRINCIPIO DE AHIMSA ${ }^{6}$ Y LA ÉTICA GLOBAL}

Actualmente los países deben enfrentar y estar preparados a cambios, movimientos, desajustes, crisis, todo en un ambiente económico, político, financiero, y del medio ambiente, por lo tanto necesita de una ética global, que influye en el nuevo modelo de la globalización, ciencia, educación, política, diálogo de culturas y religiones.

Una empresa con Ética Global puede incrementar el negocio, fidelizar los clientes y empleados, mejorar las relaciones con los proveedores y socios. Es así, que los nuevos modelos de globalización deben tener presente el entorno y culturas en cuanto a: libre mercado basado en los principios de ética global, respetando el medio ambiente y la humanidad, hacia un capitalismo espiritual.

El principio de Ahimsa es quizás uno de los principios más poderosos y hermosos de la humanidad, según Pontara (1996):

Ahimsa significa no-violencia hacia la vida, pero tiene un significado mucho más amplio. Significa también que uno no puede ofender a otra persona, debiendo compadecerse del otro, incluso si se trata de un enemigo. Para aquellos que siguen esta doctrina, no hay enemigos. Quien cree en la eficacia de esta doctrina halla el último estado, cuando se alcanza la meta, viendo el mundo a sus pies. Si expresamos nuestro amorahimsa - de tal modo que marque para siempre a nuestro enemigo, dicho enemigo nos devolverá ese amor.

6. Término sánscrito que se refiere a un concepto religioso que aboga por la no-violencia y el respeto a la vida. Es lo contrario a la himsa o daño. Habitualmente se interpreta como símbolo de paz y respeto hacia los seres capaces de sentir. La Ahimsa es una importante doctrina del Hinduismo, Jainismo, y del Budismo. 
En palabras de Mahatma Gandhi: "la vida de Ramakrishna ha sido una lección de Ahimsa (no violencia). No sabía que su amor tiene límites, geográficas o de otro tipo. Su amor divino puede ser una inspiración para todos". Todas las religiones comparten el principio de la no violencia. Tal vez el jainismo ha desarrollado este concepto más que otras religiones, es la tercera religión más importante de la India. Los Jains definen Ahimsa como reverencia a todas las formas de vida.

\section{AHIMSA: APLICANDO LOS PRINCIPIOS DE ZOROASTRO}

Cualquier pensamiento Zoroastriano parte del principio de que toda empresa debe practicar Ahimsa. Por ejemplo: Todos los planes de marketing y negocios deben ser diseñados bajo visión Ahimsa, pueden desarrollar productos para toda la humanidad, no sólo para los segmentos con alto poder adquisitivo, y política de precio justo

La religión de Zoroastro es considerada en muchas formas al Cristianismo (Smart, 1989). Ambas creen en un solo Dios, el cual creó el mundo. El culto monoteísta a Zaratustra (o Zoroastro), que cuenta con unos 40.000 practicantes en Irán, ha atravesado unos 3.200 años de historia marcados por represiones y conversiones forzadas.

Con relación a esta creencia, se tomaron cuatro organizaciones que aplican su fe, en beneficio de su población y sociedad mundial, algunos de los más representantes son:

Instituto de suero en la India - Director Cyrus S. Poonawalla (Zoroastro).

Una de las mayores compañías farmacéuticas del mundo (vacunas infantiles). Su director el doctor Poonawalla indica en la visión de la organización "salud para toda la humanidad con vacunas asequibles, a un precio bajo, está revolucionando el mundo salvando a millones de niños en todo el mundo". El 50 por ciento de los niños del mundo fueron vacunados con la vacuna de PoonaWalla.

El doctor Cyrus fiel practicante Zoroastro, se comprometió en reducir el precio de la vacuna en un 60 por ciento y hacerla disponible a la comunidad mundial a un precio de 1,75 dólares por dosis.

Grupo Godrej - Presidente Adi Godrej. El grupo Godrej es un conglomerado indio con sede en Mumbai, Maharashtra, India, en gran parte propiedad de la familia Godrej. Fue fundada en 1897 por Ardeshir Godrej y Pirojsha Godrej y opera en sectores tan diversos como el

7. Zoroastro o Zarathustra, castellanizado Zaratustra, es el nombre del profeta fundador del mazdeísmo. Se sabe poco o nada de él de manera directa, y las pocas referencias que se conocen están rodeadas de misterio y leyenda. 
inmobiliario, productos de consumo, ingeniería industrial, electrodomésticos, muebles, seguridad y productos agrícolas. Adi Godrej, presidente del grupo y uno de los hombres más ricos de la India, señala "los negocios no es sólo sobre los ingresos y las ganancias, también son para ayudar a mantener nuestra tierra verde, nuestras comunidades saludables y ayudar a niños a ser inteligente".

Grupo Tata - Presidente Cirus Pallonji Mistry. Fundada en 1868 por Jamsetji Tata. El grupo Tata ha sido llevado por miembros de la familia de Tata, los cuales son todos Zoroastrianos.

Figura 3. Una Sección de la Familia TATA

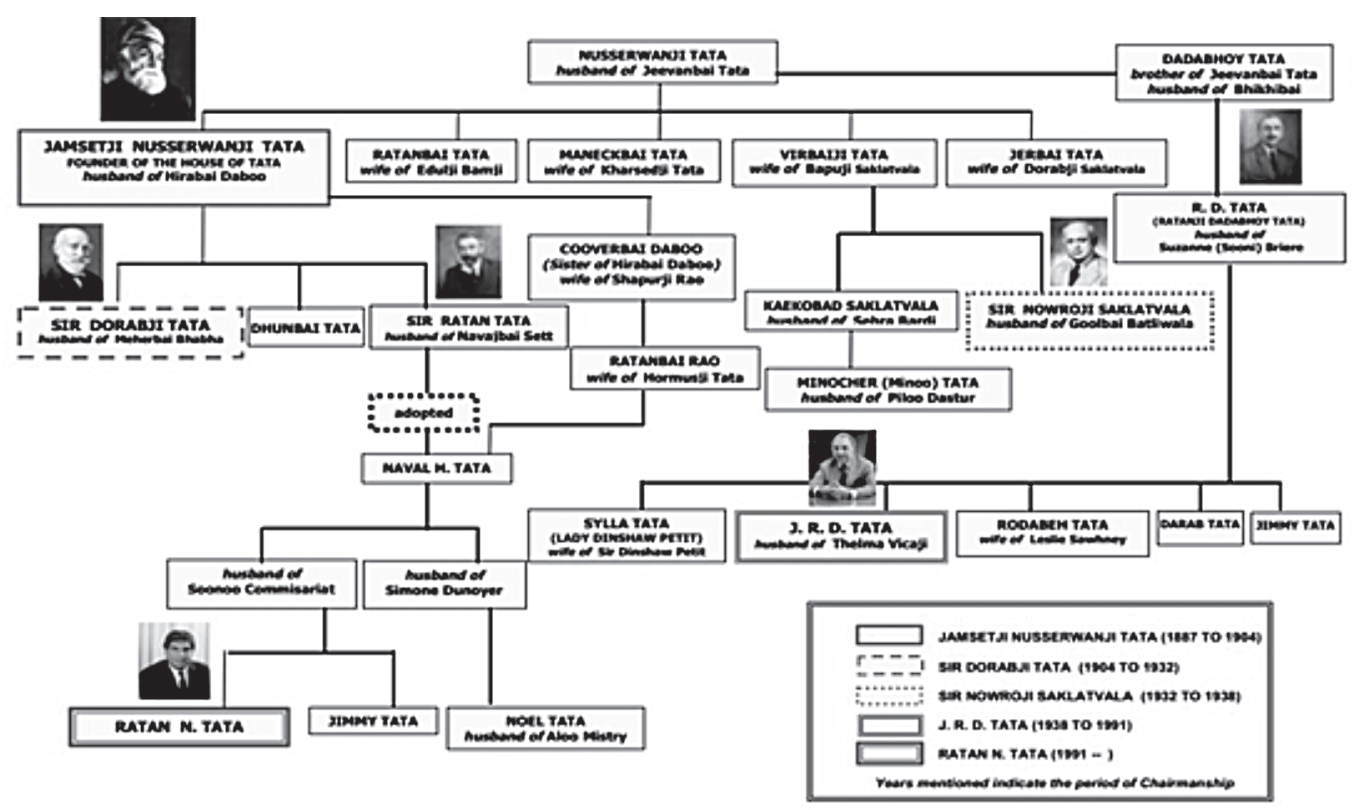

Fuente: Kmhouseindia (s.f.)

El grupo Tata se compone de más de 100 empresas operadoras en siete sectores: tecnología de información y comunicaciones, ingeniería, materiales, servicios, energía, productos de consumo y productos químicos.

La marca Tata, basada en valores de Zoroastrianos, principios sólidos y sólida base ética, es muy admirado durante sus casi 150 años de existencia.

Dice el código de conducta de Tata (1998):

Todos los empleados de una empresa de Tata, incluyendo directores de tiempo completo y el jefe del Ejecutivo, deberán exhibir comportamiento culturalmente apropiado en los países que operan en y ocuparse en nombre de la empresa con profesionalismo, 
honestidad e integridad, mientras cumple con altos estándares morales y éticos. Tal conducta será justa y transparente y percibirse a serlo por parte de terceros. Todos los empleados de una empresa Tata deberán preservar los derechos humanos de cada individuo y la comunidad y contenderá con honores compromisos.

Uno de los principios del grupo Tata es filantropía dedica el tres por ciento de sus beneficios a causas filantrópicas. Entre los años 2010-2011, el grupo Tata dedico cerca de 170 millones de dólares.

Con relación a los representantes de los Sijismos - Sikhs, esta religión combina las doctrinas del hinduismo y del islamismo, estos creyentes aplican su dogma en beneficio de su comunidad. Se presentan algunos ejecutivos y hombres de negocios que ejercen cargos en organizaciones y países como Estados Unidos, India, Canadá, Hong Kong, Kenia, Singapur, etc., dedicando importantes recursos a la filantropía como son:

- $\quad$ Ajaypal Singh Banga. Presidente y Gerente General de MasterCard. Fue nombrado por The Economic Times of India como el cuarto "indio más poderoso" del mundo.

- Malvinder y Shivinder Singh. Heredó las acciones familiares (35,5 \%) de la mayor empresa farmacéutica Ranbaxy Laboratories de la India. Los hermanos vendieron la empresa a Daiichi Sankyo de Japón por \$ 2 billones.

- Isher Judgez Ahluwalia. Presidente de la Junta de Gobernadores y el Indian Council for Research on International Economic Relations.

- Naringer Singh Kapany. Narinder Singh Kapany. Es considerado el inventor de la fibra óptica. Ha sido el Presidente fundador y principal fuente de financiación de la Fundación "Sikh".

- Joginder Singh. Jefe del estado mayor del ejército indio (2005 - 2007), el primer Sikh para dirigir al ejército indio.

\section{LA RESPONSABILIDAD SOCIAL CORPORATIVA}

Muchas compañías aplican la RSC como una mera declaración de propósitos, particularmente en los países occidentales, otros porque están realmente convencidos de devolverle parte de la sociedad de lo que ha dado, y hay otros, no muchos, que incluye la Responsabilidad Social Empresarial (RSE), como una parte fundamental de su estrategia de negocio, directamente influyen en su producto y servicio de estrategia de desarrollo y precio.

La RSC según Fernández (2009) transmite la visión de una empresa hacia la sociedad y el medio ambiente. Si la sociedad ha permitido a la compañía desarrollar su negocio, la compañía, de alguna manera, está en deuda con la sociedad, más allá de los requisitos legales vigentes.

De esta forma, la RSC transmite los principios éticos y los valores de la empresa, para que esto sea posible, la lucha contra la corrupción y la defensa de los derechos humanos debe ser parte de la RSE de la compañía. 
A continuación se presentan algunos de sus representantes y sus contribuciones a la sociedad.

- $\quad$ Alhaji Aliko Dangote (Grupo Dangote Nigeria), es el hombre más rico de África, musulmán sunita. Dangote ha donado 100 millones de dólares de su fortuna a obras de caridad en los últimos años.

- Vladimir Potanin Presidente (Interros - Vladimir Potanin Foundation), es un Filántropo y empresario ruso, es un cristiano ortodoxo. El doctor Potain en 2010, hizo un compromiso público para dar a la mayoría de su fortuna a causas filantrópicas.

- $\quad$ Amancio Ortega (Grupo Inditext Zara -España), es el cuarto hombre más rico del mundo. En sus empresas tiene unos 120,000 empleadores. Cristiano católico. A través de su fundación en octubre de 2012 donó 20 millones de euros a Cáritas.

Otros líderes espirituales y religiones están mostrando el camino. Algunas empresas líderes mundiales están desarrollando sus enseñanzas como son:

Bhavarlal Hiralal Jain, es el Director de sistemas de irrigación Jain. Fue pionero en el concepto de micro riego en la India. Jainismo. La misión de él es: "Dejar este mundo mejor que lo encontró". Bhavarlal Hiralal Jain señala que

el crecimiento en términos de volumen de ventas y valor neto no es la única preocupación de Jain. Con el paso del tiempo y el progreso material, los Jainistas han continuado creciendo sus horizontes filantrópicas. No hay límites para el sufrimiento humano y la generosidad humana.

Carlos Slim, es el segundo hombre más rico del mundo (Forbes en su informe de 2015). Maronita cristiano. Fundador del Grupo Carso (78,000 empleados). Uno de los filántropos más grandes del mundo. En 2007, la Fundación Carlos Slim donó USD 100 millones para luchar contra la pobreza en América Latina; en 2008, Carlos Slim Helú dona USD\$ 110 millones para ayudar a los niños americanos; en 2009, Muhammad Yunus (Fideicomiso de Grameen de Bangladesh, musulmán) y Carlos Slim lanzamiento del proyecto Grameen-Carso (USD\$ 45 millones). Y en 2013 Carlos Slim anunció que le donan USD\$ 100 millones a la iniciativa mundial para la erradicación de la poliomielitis

\section{AGNÓSTICOS}

Bill \& Melinda Gates, dicen "cada vida tiene igual valor". Tanto Bill Gates (una fortuna de 79.2 mil millones de dólares) como Warren Buffet (72.2 mil millones de dólares) tiene la misma visión filantrópica de "retorno a parte de la sociedad de lo nos ha dado".

Buffet se había comprometido a donar el 99 por ciento de su fortuna a la Fundación Bill y Melinda Gates. La iniciativa filantrópica más grande del oeste, el compromiso de dar fue 
creada en 2010 por multimillonarios Warren Buffett y Bill Gates, los agnósticos, con el objetivo de que los estadounidenses más ricos donen al menos un 50 por ciento de su fortuna a obras de caridad. Se estima que desde su creación \$125 mil millones de dólares ya se han comprometido. Esta iniciativa está compuesta por agnósticos y por personas de diferentes religiones: cristianos (católicos, protestantes, metodistas y mormones), los judíos, hinduista, musulmanes, entre otras religiones.

\section{CONCLUSIÓN}

Ahora bien, crear negocio para las organizaciones se vuelve cada vez más complicado por diversos factores entre ellos la competencia y es aún más complejo sí se habla de la escasa confianza al realizar transacciones nacionales a un más internacionales. Lo anterior, porque cada nación tiene desiguales formas de hacerlo.

Las personas encuentran en la religión un motivo de ser y justicia, pero al definir a la religión se requiere la inclusión de lo sobrenatural y la existencia de un poder más profundo. La religión define los ideales de la vida, que a su vez es reflejada en los valores y actitudes de sociedades e individuos. Tales valores y actitudes conforman el comportamiento y las prácticas de las personas, Como expone la Morales (2013):

La religión es una influencia de las más grandes en los negocios internacionales, llámese como se llame la religión; budismo, cristianismo, hinduismo, islamismo y judaísmo, etc., cuyas creencias específicas suelen afectar los negocios internacionales si no se conocen, por ejemplo, algunos grupos cristianos prohíben el consumo de bebidas alcohólicas, pero otros no. También las diferencias entre naciones practicantes de la misma religión llegan a tener efectos en los negocios internacionales. Por lo general, el viernes no es un día hábil para los negocios en los países musulmanes, ya que se dedica ese día al culto; sin embargo, han adoptado el calendario laboral cristiano para garantizar tratos más productivos como Europa.

Otro aspecto fundamental, es el cumplimiento de las creencias de cada religión en beneficio de la humanidad, ya se evidenciaron ampliamente un sin número de ejemplo de organizaciones y personajes creyentes de su fe, y el éxito que presentan sin interés (filantropía), para beneficio de una sociedad mundial necesitada de estos sustentos.

En la actualidad la ética global empiezan a difundirse a todos los lugares de la tierra. La ética, entendida como esa disciplina cuyo objetivo es lograr el bien entre los hombres, moviendo las empresas a buscar de una manera fundamental que son personas las que deberían promover la ética y no al contrario. La ética global en el comercio internacional no se puede cambiar para mejorar, si no se cambia la conciencia de la gente en busca de comprensión y mutuo respeto entre los participantes. 
Siglos atrás las religiones ya ofrecieron normas de conducta y valores para que las personas implementen en su cotidiano vivir y más aún al libre comercio local e internacional. Por esta razón las personas de diferentes dogmas (hindú, cristiana, islámica, budistas, etc.) anhelan conocer cómo implementar sus creencias para el entorno empresarial para una mejor comprensión entre las partes y crear sinergias entre ellas (religión, cultura y comercio).

En las transacciones comerciales entre diversos dogmas, hoy debe existir interacción religiosa y conocimiento de las mismas. Por lo tanto, ignorar o malinterpretan las creencias puede causar serios problemas culturales e incluso con las supresiones de transacciones, negocios, o convenios importantes.

Sobre el cristianismo, religión que tiene aproximadamente 2000 millones fieles, estos practicantes abogan por un sentido de justicia en los negocios, como el correcto cumplimiento en los contratos que se llevan a cabo, la adherencia a las normas y trabajo en pos de la común bien. El Islam, con más de 1000 millones fieles, (según el informe de Pew Center), es uno de los cultos más importantes del mundo y continúa creciendo, varios estudiosos en el campo de la ética empresarial perteneciente al Islam de acuerdo como un concepto central de esta religión, en donde las organizaciones deben maniobrar y que debe hacerse según las leyes de la naturaleza emanadas por Dios.

Comprender cómo se definen las diferentes religiones del mundo e incluso los no creyentes (agnósticos) es fundamental para fomentar la tolerancia, el entendimiento mutuo y un mayor conocimiento para el manejo de las negociaciones internacionales, así entonces, podría conducir a una mayor comprensión de las sociedades del mundo y promover el diálogo entre las diferentes religiones existentes. Sin embargo, la relación entre la economía y la religión está basada en un reconocimiento de la interdependencia de ambas, de cómo la Iglesia reconoce que las creencias de las personas tienen un impacto sobre el desarrollo de la economía y a su vez la economía interviene sobre el comportamiento de las personas, razón por la cual ambas pueden ser consideradas como sistemas de valores éticos. Ésta visión cada vez es más aceptada por la Iglesia Católica, de ahí que la vertiente social de ésta ha emitido desde el año de 1891 con el Papa León XIII y su famosa encíclica Rerum Novarum, documentos relativos al contexto de la vida económica.

En lo referente, al perfil del líder o gestor de comercio internacional en una organización, este debe ser una persona ética, preparada y dinámica para un mundo globalizado que hoy requiere de profesionales cualificados, calificados y emprendedores, con experiencia y educación en negocios internacionales. Él o ella necesitan estar en continua capacitación acerca de Incoterms, despacho de aduana, distribución internacional, los precios, adaptación de productos, las políticas de promoción y negociación intercultural. El idioma preferiblemente debe dominar dos o tres idiomas (Inglés, Francés, Árabe o incluso los chinos); también y aspecto fundamental, un conocimiento y respeto a las religiones del mundo. Si deben viajar a África, Asia o el Medio Oriente, se recomienda el conocimiento y respeto al Islam. Por otra 
parte cuando corresponda realizar negocios en Asia entonces se recomienda, conocimiento del budismo, el confucianismo y el taoísmo. Cuando un país como la India es un país clave para el mercado de su organización, deben conocer de hinduismo, jainismo, Sijismo y zoroastrismo y finalmente, abrir la mente y el corazón.

\section{REFERENCIAS}

Aureoles, K. (2012). Cultura y negocios internacionales. Recuperado de https://prezi.com/ xxn6yipfrkta/cultura-y-negocios-internacionales/

Ball, D. \& Culloch W. (1997). Negocios internacionales. Introducción y aspectos esenciales. México: Mc Graw Hill.

Fernández, R. (2009). Responsabilidad social corporativa. Alicante: Editorial Universitaria.

Gandhi, M. (1930). Rethinking the possibility of Non-violence. Recuperado de http://www. mkgandhi.org/articles/nonviolence1.htm

Global Ethics, Religion and International Business (2013). EENI. Recuperado de http:// en.reingex.com/Religion-Ethics-Business.shtml

Lee, C. (2013). Does religion affect international trade in services more than trade in goods? Applied Economics Letters, 20(10), 998-1002, doi: 10.1080/13504851.2013.770120

Lenski, G. (1967). El factor humano, Barcelona: Labor.

Los Efectos de la Religión en la Economía. (2006). Redes cristianas. Recuperado de: http:// www.redescristianas.net/2006/08/21/los-efectos-de-la-religion-en-la-economia/

Morales, E. (2013). La Religión y los Negocios Internacionales. Recuperado de: http:// lareligionylosnegociosinternacionales.blogspot.com/

Molero, V. (2006). Generación marketing. La sociedad entre la codicia y la indolencia. Madrid: Esic.

Nonell, P. (2013). Ética global, religiones y negocios internacionales. Recuperado de: http:// www.reingex.com/Religion-Etica-Negocios.shtml

Pontara, G. (1996). Introducción. En M.K. Gandhi, Teoria e pratica della non-violenza. Torino: Einaudi. 
Smart, N. (1989). Las religiones del mundo. Madrid: Akal.

Sri Ramakrishna (1903), The Sayings of Sri Ramakrishna New York: The Vedanta Society, Recuperado de: http://www.estudantedavedanta.net/Sayings\%20of\%20Sri\%20 Ramakrishna.pdf

Status of women on Sikhism, (2005). SikhWomen.com Recuperado de: http://www.sikhwomen. com/Guru_Quotes/women.htm

Tata (1998). Ethical conducts Claude Recuperado de: http://www.tata.com/aboutus/ articlesinside/Tata-Code-of-Conduct 